\title{
Sensitivity on anomalous neutral triple gauge couplings via $Z Z$ production at FCC-hh
}

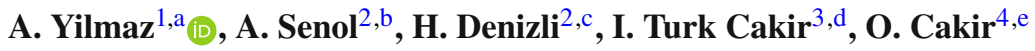 \\ ${ }_{1}^{1}$ Department of Electrical and Electronics Engineering, Giresun University, 28200 Giresun, Turkey \\ ${ }^{2}$ Department of Physics, Bolu Abant Izzet Baysal University, 14280 Bolu, Turkey \\ ${ }^{3}$ Department of Energy Systems Engineering, Giresun University, 28200 Giresun, Turkey \\ ${ }^{4}$ Department of Physics, Ankara University, 06100 Ankara, Turkey
}

Received: 14 June 2019 / Accepted: 10 February 2020 / Published online: 24 February 2020

(C) The Author(s) 2020

\begin{abstract}
We study the sensitivity of anomalous neutral triple gauge couplings $(a N T G C$ ) via $p p \rightarrow Z Z$ production in the $4 \ell$ channel at $100 \mathrm{TeV}$ center of mass energy of future circular hadron collider, $\mathrm{FCC}-\mathrm{hh}$. The analysis including the realistic detector effects is performed in the mode where both $\mathrm{Z}$ bosons decay into same-flavor, oppositely charged lepton pairs. The sensitivities to the charge-parity (CP)-conserving couplings $C_{\tilde{B} W} / \Lambda^{4}$ and CP-violating couplings $C_{W W} / \Lambda^{4}$, $C_{B W} / \Lambda^{4}$ and $C_{B B} / \Lambda^{4}$ obtained at $95 \%$ Confidence Level using the invariant mass distribution of the $4 \ell$ system reconstructing the leading and sub-leading $\mathrm{Z}$ boson candidates are $[-0.09,+0.09],[-0.21,+0.21],[-0.26,+0.26]$, and $[-0.10,+0.10]$ in units of $\mathrm{TeV}^{-4}$, respectively.
\end{abstract}

\section{Introduction}

The studies on the diboson production at colliders play an important role in testing the non-Abelian SU $(2)_{L} \times U(1)_{Y}$ gauge group of the electroweak sector in the Standard Model (SM) and searching for new phenomena at the TeV-energy scale [1]. Since there are no triple gauge couplings between the photon and $Z$ boson $(Z \gamma \gamma$ and $Z \gamma Z)$ at tree level except $W W Z$ and $W W \gamma$ in the SM. Therefore any deviations from SM predictions on neutral triple gauge (NTG) couplings (including $Z Z \gamma, Z \gamma \gamma$ and $Z Z Z$ vertices) can give an indication about new physics beyond the SM. The new physics effects at high energy can be parametrized in the Effective Field Theory (EFT) approach. This approach is gen-

\footnotetext{
a e-mail: aliyilmaz@ giresun.edu.tr (corresponding author)

b e-mail: senol_a@ibu.edu.tr

c e-mail: denizli_h@ibu.edu.tr

de-mail: ilkay.turk.cakir@cern.ch

e e-mail: ocakir@science.ankara.edu.tr
}

eral enough to point out the most probable places to observe these effects; also it includes the gauge symmetries of the standard model and can be used at both tree level and loop level. Anomalous NTG vertices can be added in an effective Lagrangian using the EFT approach and parametrized by $\mathrm{CP}$-conserving and $\mathrm{CP}$-violating couplings, while no electroweak NTGC is present at tree level [2].

The production of $Z Z$ dibosons leading to the $4 \ell$ final state has been studied by various collaborations such as the Large Electron-Positron (LEP) collaboration [3-8] where the first bounds on anomalous neutral triple gauge couplings (aNTGCs) using $\mathrm{e}^{+} \mathrm{e}^{-}$collider were obtained, the Collider Detector at Fermilab (CDF) collaboration $[9,10]$ and $D \emptyset$ collaborations $[11,12]$ also researched the limits of aNTGC at the Tevatron $p \bar{p}$ collider. Recently, the ATLAS $[13,14]$ and CMS $[14,15]$ collaborations published the improved limits of aNTGCs using the center of mass energy of LHC of 13 $\mathrm{TeV}$. This high center of mass energy leads to enhancement of the cross-section, which would widen the range of triple gauge coupling studies. There are also some phenomenological studies probing the sensitivities of aNTGCs at hadron colliders in the EFT framework [16-20].

The dimension-eight (dim-8) effective Lagrangian for nTGC in the scope of EFT assuming the local U(1) $E M$ and Lorentz symmetry can be written as [21]

$\mathcal{L}^{n T G C}=\mathcal{L}_{S M}+\sum_{i} \frac{C_{i}}{\Lambda^{4}}\left(\mathcal{O}_{i}+\mathcal{O}_{i}^{\dagger}\right)$

where $i$ is the index of equations running over the operators given as

$$
\begin{aligned}
\mathcal{O}_{\widetilde{B} W} & =i H^{\dagger} \widetilde{B}_{\mu \nu} W^{\mu \rho}\left\{D_{\rho}, D^{\nu}\right\} H, \\
\mathcal{O}_{B W} & =i H^{\dagger} B_{\mu \nu} W^{\mu \rho}\left\{D_{\rho}, D^{\nu}\right\} H, \\
\mathcal{O}_{W W} & =i H^{\dagger} W_{\mu \nu} W^{\mu \rho}\left\{D_{\rho}, D^{\nu}\right\} H, \\
\mathcal{O}_{B B} & =i H^{\dagger} B_{\mu \nu} B^{\mu \rho}\left\{D_{\rho}, D^{\nu}\right\} H,
\end{aligned}
$$


where $\widetilde{B}_{\mu \nu}$ is the dual $B$ strength tensor. We used the convention in the definitions of the operators of

$B_{\mu \nu}=\left(\partial_{\mu} B_{\nu}-\partial_{\nu} B_{\mu}\right)$,

$W_{\mu \nu}=\sigma^{I}\left(\partial_{\mu} W_{\nu}^{I}-\partial_{\nu} W_{\mu}^{I}+g \epsilon_{I J K} W_{\mu}^{J} W_{\nu}^{K}\right)$,

with $\left\langle\sigma^{I} \sigma^{J}\right\rangle=\delta^{I J} / 2$ and

$D_{\mu} \equiv \partial_{\mu}-i g_{w} W_{\mu}^{i} \sigma^{i}-i \frac{g^{\prime}}{2} B_{\mu} Y$.

If the new physics energy scale is high, the largest new physics contribution to the $f f \rightarrow Z Z$ process is expected from the interference between the SM and the dim- 8 operators,

$$
\begin{aligned}
|M|^{2}= & \underbrace{\left|M_{S M}\right|^{2}}_{\mathcal{O}\left(\Lambda^{0}\right)}+\underbrace{2 \Re\left(M_{S M} M_{d i m-8}^{*}\right)}_{\mathcal{O}\left(\Lambda^{-4}\right)} \\
& +\underbrace{2 \Re\left(M_{S M} M_{d i m-10}^{*}\right)}_{\mathcal{O}\left(\Lambda^{-6}\right)} \\
& +\underbrace{\left|M_{d i m-8}\right|^{2}+2 \Re\left(M_{S M} M_{d i m-12}^{*}\right)}_{\mathcal{O}\left(\Lambda^{-8}\right)}+\mathcal{O}\left(\Lambda^{-10}\right) .
\end{aligned}
$$

The square of the amplitude with dim-8 operators $\left(\left|M_{\operatorname{dim} 8}\right|^{2}\right)$ is given to understand the suppression of the interference $\left(2 \Re\left(M_{S M} M_{\operatorname{dim} 12}^{*}\right)\right)$ but it does not induce a leading contribution from the heavy new physics unless the interferences between the SM and the dim-8 $\left(2 \Re\left(M_{S M} M_{d i m 8}^{*}\right)\right)$ and dimension-ten (dim-10) operators $\left(2 \Re\left(M_{S M} M_{\text {dim } 10}^{*}\right)\right)$ are both strongly suppressed. The dim- 6 operators can have an effect on nTGC at one-loop level (at the order $\mathcal{O}\left(\alpha \hat{s} / 4 \pi \Lambda^{2}\right)$ as they do not induce nTGC at tree level [21]. However, the tree-level contributions from dim-8 operators are of the order $\mathcal{O}\left(\hat{s} v^{2} / \Lambda^{4}\right)$. Therefore, a one-loop contribution of the dim- 6 operators can be ignored with respect to that of dim-8 operators for $\Lambda \lesssim 2 v \sqrt{\pi / \alpha}$.

The coefficients of these four dim-8 operators describing aNTGC are the CP-conserving couplings $C_{\tilde{B} W} / \Lambda^{4}$ and CP-violating $C_{W W} / \Lambda^{4}, C_{B W} / \Lambda^{4}$ and $C_{B B} / \Lambda^{4}$. The current limits on the couplings $C_{\tilde{B} W} / \Lambda^{4}, C_{W W} / \Lambda^{4}, C_{B W} / \Lambda^{4}$ and $C_{B B} / \Lambda^{4}$ are given in Table 1 . These are couplings of the dim- 8 operators converted from the couplings of the dim- 6 operators for the process $p p \rightarrow Z Z \rightarrow \ell^{+} \ell^{-} \ell^{\prime+} \ell^{\prime-}$ [14]. Here $\ell=e$ or $\mu$ and $Z \gamma \rightarrow \nu \bar{v} \gamma$ [22] at the center of mass energy $\sqrt{s}=13 \mathrm{TeV}$ and integrated luminosity $L_{\text {int }}=36.1$ $\mathrm{fb}^{-1}$ originating from the LHC. In this table, all couplings other than the one under study are set to zero.

In the future circular collider project, FCC [23], one has proposed to have three collider options (FCC-ee, FCC-eh and FCC-hh) working at different center of mass energies. The hadron collider option of FCC (FCC-hh) is planned to reach an integrated luminosity of $20-30 \mathrm{ab}^{-1}$ at $100 \mathrm{TeV}$
Table 1 Observed one dimensional 95\% confidence level (C.L.) limits on $C_{\tilde{B} W} / \Lambda^{4}, C_{W W} / \Lambda^{4}, C_{B W} / \Lambda^{4}$ and $C_{B B} / \Lambda^{4}$ EFT parameters from LHC

\begin{tabular}{lll}
\hline $\begin{array}{l}\text { Couplings } \\
\left(\mathrm{TeV}^{-4}\right)\end{array}$ & Limit 95\% C.L. & \\
\cline { 2 - 3 } & $Z Z \rightarrow 4 \ell[14]$ & $Z \gamma \rightarrow v \bar{v} \gamma[22]$ \\
\hline$C_{\tilde{B} W} / \Lambda^{4}$ & $-5.9,+5.9$ & $-1.1,+1.1$ \\
$C_{W W} / \Lambda^{4}$ & $-3.0,+3.0$ & $-2.3,+2.3$ \\
$C_{B W} / \Lambda^{4}$ & $-3.3,+3.3$ & $-0.65,+0.64$ \\
$C_{B B} / \Lambda^{4}$ & $-2.7,+2.8$ & $-0.24,+0.24$ \\
\hline
\end{tabular}

center of mass energy. FCC-hh, comparing to LHC, has the energy scale enhanced by a factor about 7 depending on the process [24].

Exploring the new physics effects in the production of a diboson is a challenging task. In the literature $\mathrm{ZZ}$ diboson production has been examined in two decay channels, such as the " $2 \ell 2 v$ " and the " $4 \ell$ " channel [2]. In the first channel, $Z Z \rightarrow 2 \ell 2 v$, one of the $Z$ decays into a sameflavor, oppositely-charged two leptons, while the other one decays into neutrinos, which leads to an increase in the missing transverse energy in the final state. Therefore this channel is exposed to a larger background contribution and it is not kinematically reconstructable completely. In the second decay channel, $Z Z \rightarrow 4 \ell$, not only the first $Z$ boson, but also the other $Z$ boson decays into two same-flavor, oppositely charged leptons. This process gives rise to the inclusion of a very low background, being kinematically reconstructable in the final state. On the other hand, one needs to take into account that the process has small branching fractions resulting in a low statistics in the final state.

This paper will be organized as follows: In Sect. 2 we will discuss the simulation environment of $Z Z$ diboson production for signal and background at the FCC-hh collider. Event selection procedures of our phenomenological study in the $4 \ell$ final state will be given in Sect. 3. In Sect. 4, we will give the collected results for the $4 \ell$ final state analysis. Conclusions on the sensitivities of each coupling will be summarized in Sect. 5.

\section{Generation of signal and background events}

The purpose now was to obtain the bounds on aNTGC parameters of $Z Z$ diboson production in the framework of the EFT at the FCC-hh. We generated signal and background events for the $p p \rightarrow Z Z$ process by importing the signal aTGC implemented through the UFO model file into MadGraph5_aMC@NLO v2.6.4 [25]. The PYTHIA V8.2 [26] package is used for parton showering and hadronization. The LHAPDF $v 6.1 .6$ [27] library and its NNPDF $\quad$ 2 2.3 [28] set is used as the default set of parton 


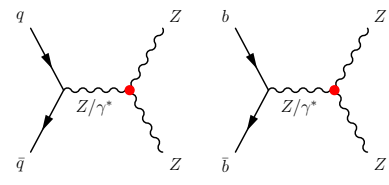

(a) signal

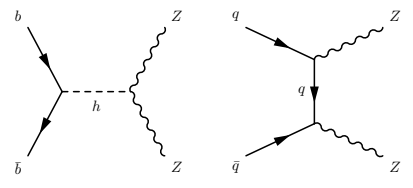

(b) background
Fig. 1 The leading order Feynman diagrams of ZZ production a for signal including an aNTGC vertex depicted by a red dot, $\mathbf{b}$ for the SM background

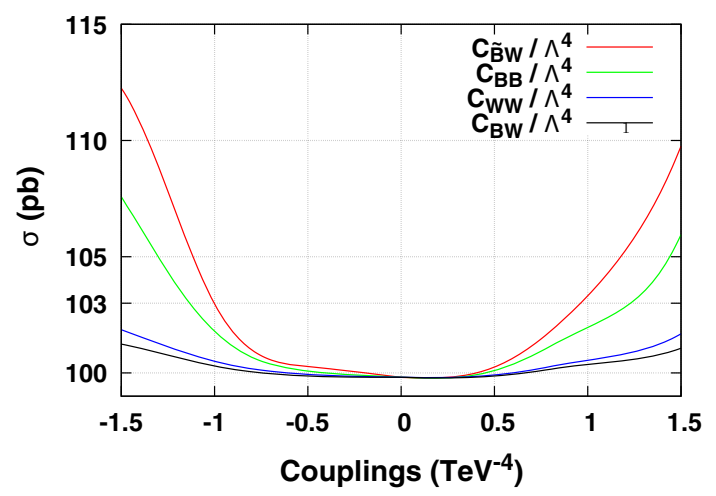

Fig. 2 Cross-sections for the process with aNTGCs including CPconserving and $\mathrm{CP}$-violating terms in the Lagrangian

distribution functions (PDFs) for all simulated MC samples. There were generated $3 \times 10^{6}$ events of the signal and the background for each of the dim-8 couplings. The detector response is simulated using a detailed description of the FCChh detector card implemented in Delphes v3.4.1 [29]. All events are analyzed by using the ExRootAnalysis [30] package with ROOT V6.16 [31]. The kinematical distributions are normalized to the number of expected events, which is defined to be the cross-section of each process including the branching times integrated luminosity of $\mathcal{L}_{i n t}$ $=10 \mathrm{ab}^{-1}$.

The leading order Feynman diagrams that contribute to the signal and its main-background processes are shown in Fig. 1a, b, respectively. The red dot represents the aNTGC vertex in the production of $Z Z$.

The cross-sections of the $Z Z$ process in the generator level as a function of the four dim-8 couplings mentioned are shown in Fig. 2 where the default mass of the $Z$ boson is chosen as $91.187 \mathrm{GeV}$. In this figure, only one coupling at a time is varied from its SM value and plotted as a function of the couplings in the range of limits reported by the CMS collaboration [15]. One can clearly see the deviation from the SM in Fig. 2. In the analysis, we include effective dim-8 aNTG operators and an SM contribution as well as interference between effective operators and SM contributions. A slightly asymmetric behavior from Fig. 2 is seen and the contributions from both $C_{i} / \Lambda^{4}$ and $\left(C_{i} / \Lambda^{4}\right)^{2}$ terms appear to be suppressed for values of the $C_{i} / \Lambda^{4}$ couplings in the range $[-1.5,1.5] \mathrm{TeV}$. The interference contributions $\left(\sim C_{i} / \Lambda^{4}\right)$ are quite suppressed compared to the dim-8 squared terms.

\section{Event selection}

We consider the $4 \ell$ final state in our analysis based on Ref. [15] including three possible options: $e^{+} e^{-} e^{+} e^{-}$, $\mu^{+} \mu^{-} \mu^{+} \mu^{-}$, and $e^{+} e^{-} \mu^{+} \mu^{-}$. The preselection for this analysis requires the presence of a pair of dileptons of the same or different flavors [32]. All permutations of leptons giving a pair of $Z / \gamma^{*}$ candidates are considered within each event. The pairing ambiguity is resolved by ordering the pair of dilepton candidates based on the differences between the reconstructed invariant mass of the dilepton candidate $\left(m_{\ell^{+} \ell^{-}}\right)$and the nominal $Z$ boson mass $m_{Z}$. Therefore, the dilepton candidate with an invariant mass closest to the nominal $Z$ boson mass [33] is called leading $Z$ while the second closest is defined as sub-leading $Z$.

In the reconstructed leading $Z$ (or sub-leading $Z$ ), the lepton $\ell^{1}$ is labeled as the highest- $p_{T}$ lepton, while $\ell^{2}$ is labeled as the second highest- $p_{T}$ lepton among the pairs.

In order to see the region where the signal $\left(C_{\widetilde{B} W} / \Lambda^{4}=\right.$ 5.0 $\mathrm{TeV}^{-4}$ ) can be enhanced, we plotted the transverse momentum of leptons $\left(p_{T}^{\ell^{1}}, p_{T}^{\ell^{2}}\right)$ versus the reconstructed invariant mass of the leading and sub-leading $Z$ as shown in Figs. 3, 4 and 5 for the $4 e, 4 \mu$ and $2 e 2 \mu$ decay channels, respectively. Events with a pair of dileptons, leading $Z$ hav-

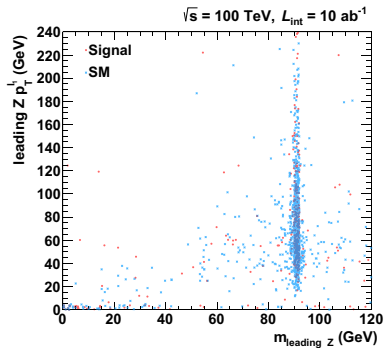

(a)

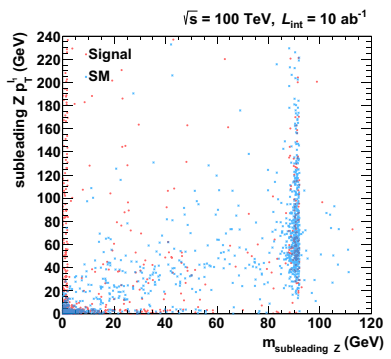

(c)

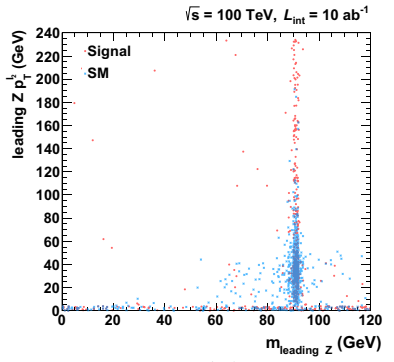

(b)

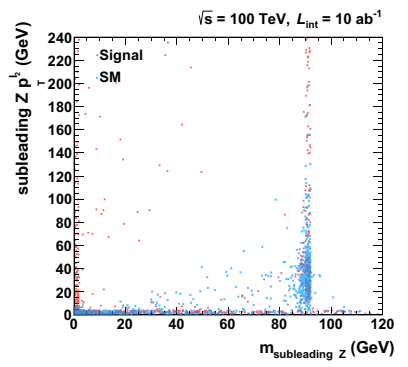

(d)
Fig. 3 Transverse momentum distributions of a leading lepton, b subleading lepton of the leading $Z$ boson vs its invariant mass and transverse momentum distributions of $\mathbf{c}$ leading lepton, $\mathbf{d}$ sub-leading lepton of the sub-leading $Z$ boson vs its invariant mass in the $4 e$ channel 


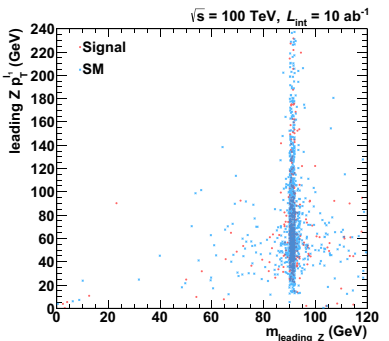

(a)

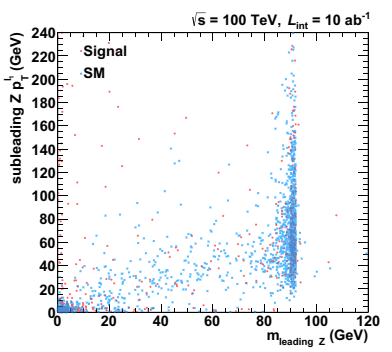

(c)

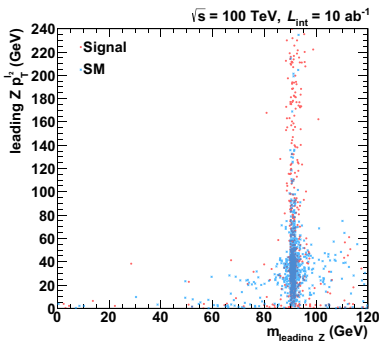

(b)

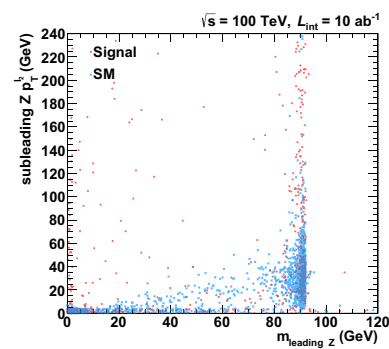

(d)

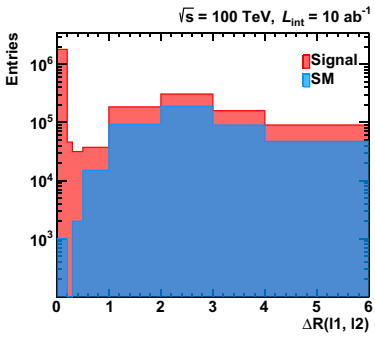

(a)

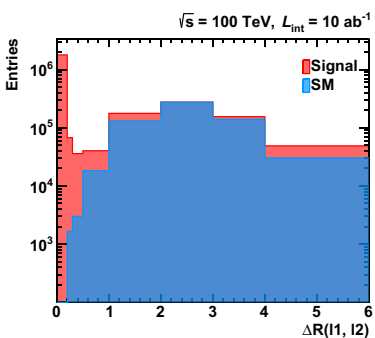

(c)

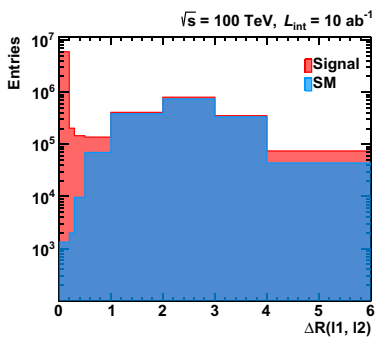

(e)

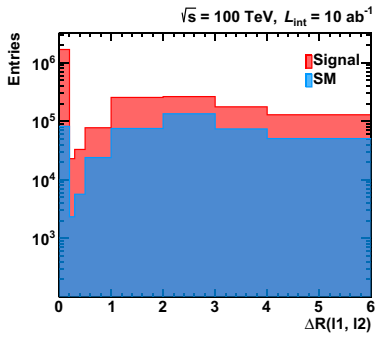

(b)

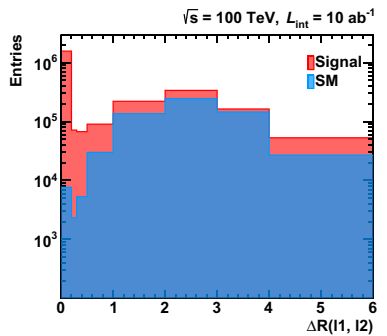

(d)

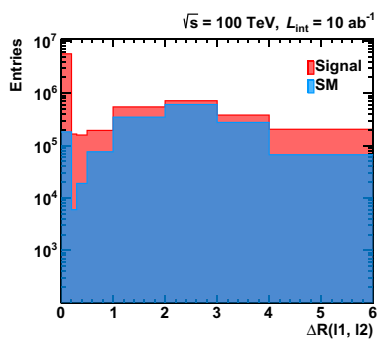

(f)
Fig. $6 \Delta R$ distributions between leptons of candidate $\mathrm{Z}$ boson pairs in the $4 e(\mathbf{a}, \mathbf{b}), 4 \mu(\mathbf{c}, \mathbf{d})$ and $2 e 2 \mu(\mathbf{e}, \mathbf{f})$ channels. a, c, e for leading $Z$ and $\mathbf{b}, \mathbf{d}$ and $\mathbf{f}$ for sub-leading $Z$ bosons

(b)

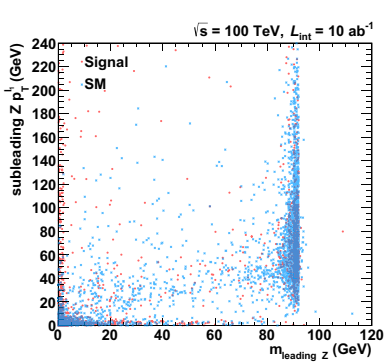

(c)

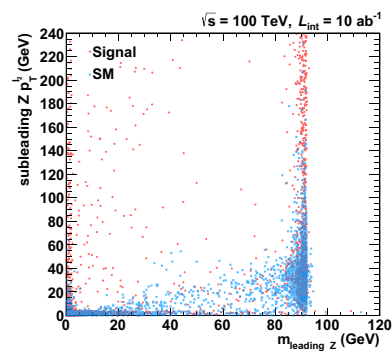

(d)
Fig. 5 Transverse momentum distributions of a leading lepton, b subleading lepton of the leading $Z$ boson vs its invariant mass and transverse momentum distributions of $\mathbf{c}$ leading lepton, $\mathbf{d}$ sub-leading lepton of the sub-leading $Z$ boson vs its invariant mass in the $2 e 2 \mu$ channel

ing $p_{T}^{\ell^{1}}>20 \mathrm{GeV}(20 \mathrm{GeV})$ and $p_{T}^{\ell^{2}}>12 \mathrm{GeV}(10 \mathrm{GeV})$ for electron (muon) and sub-leading $Z$ having $p_{T}^{\ell^{1}, \ell^{2}}>5 \mathrm{GeV}(5$ $\mathrm{GeV}$ ) for electron (muon), are selected for further analysis. These cuts are applied for both signal and background events in order to focus the nominal mass region of the $Z$ boson.
The pseudo-rapidity cuts of all leptons are applied as $\left|\eta^{\ell}\right|<2.5$. The distance $\Delta R\left(\ell^{1}, \ell^{2}\right)$ between leptons in the $\eta-\phi$ plane is evaluated by the function

$\Delta R\left(\ell^{1}, \ell^{2}\right)=\sqrt{\left(\eta^{\ell^{1}}-\eta^{\ell^{2}}\right)^{2}+\left(\phi^{\ell^{1}}-\phi^{\ell^{2}}\right)^{2}}$

and plotted in Fig. 6. The distributions between two leptons of leading and sub-leading $\mathrm{Z}$ are in the first and second column. Each row corresponds to different decay channel aligned for $4 e, 4 \mu$ and $2 e 2 \mu$, respectively. As seen in Fig. 6, the distributions are strongly peaked, $\Delta R\left(l^{1}, l^{2}\right) \sim 3$, for signal and background. This is due to the SM cross-section being ,dominated by the threshold region $\sqrt{\hat{s}} \sim 2 M_{Z}$, where the Z-boson momenta are small and the decay leptons tend to occur backto-back. One can start to see anomalous couplings effects on the cross-section in the large $\mathrm{Z}$ boson transverse momentum region. The relative opening angle between the leptons constructing the Z-boson and Z-boson transverse momentum are inversely related due to the Lorentz boost, i.e. an increase in $p_{T}^{Z}$ causes a reduction in the opening angle [34]. The devi- 
Table 2 Preselection and a set of cuts for the analysis of signal and background events

\begin{tabular}{ll}
\hline Cuts & Definition \\
\hline Cut-0 & Preselection: $N_{\ell_{(e, \mu)}} \geq 4$ and \\
& two same-flavor opposite-charge lepton pairs \\
Cut-1 & $\begin{array}{l}\text { Dileptons minimizing }\left|m_{\ell \ell}^{a}-m_{Z}\right|+\left|m_{\ell \ell}^{b}-m_{Z}\right| \text { are } \\
\text { taken as } \\
\end{array}$ \\
& Z boson pair candidates
\end{tabular}

Cut-2 Transverse momentum of a dilepton pair: $p_{T}^{\ell^{1}}>20 \mathrm{GeV}$, $p_{T}^{\ell^{2}}>12 \mathrm{GeV}(10 \mathrm{GeV})$ in the constructed leading $Z$ for $e(\mu)$ and in the constructed sub-leading $Z p_{T}^{\ell^{1,2}}>5$ $\mathrm{GeV}(5 \mathrm{GeV})$ for $e(\mu)$

Cut-3 Pseudo-rapidity: $\left|\eta^{\ell}\right|<2.5$

Cut-4 $\Delta R\left(\ell^{1}, \ell^{2}\right)>0.02$ between all leptons

Cut-5 Invariant mass: $80<M_{i n v}^{r e c}($ leading $\mathrm{Z})<100 \mathrm{GeV}$ and $60<M_{i n v}^{r e c}($ sub-leading $\mathrm{Z})<110 \mathrm{GeV}$

ations due to non-standard $Z Z V$ couplings $(V=Z$ or $\gamma$ ) in the $\Delta R\left(l^{1}, l^{2}\right)$ distributions are therefore concentrated at rather small values as seen in Fig. 6. In order to meet the detector requirement, we applied a cut for all leptons which are separated from each other by imposing $\Delta R\left(\ell^{1}, \ell^{2}\right)>$ 0.02 .

The $p p \rightarrow Z Z$ sensitivity is estimated by using events where a further cut is applied for both invariant mass of leading $Z$ and sub-leading $Z$ bosons must be within the range 80 $<m_{\text {leading } Z}<100 \mathrm{GeV}$ and $60<m_{\text {sub-leading } Z}<110$ $\mathrm{GeV}$, respectively. These ranges were chosen to keep most of the decays in the resonance, while removing most other processes with $4 \ell$ final states. Decays of the $Z$ bosons to $\tau$ leptons with subsequent decays to electrons and muons are heavily suppressed by requirements on the lepton's $p_{T}$.

The cut flow steps in the analysis for selecting the events are summarized in Table 2.

After applying the kinematical cuts discussed above, the reconstructed invariant mass of the leading $Z$ boson candidates and a contour plot showing the correlation between sub-leading $Z$ boson versus leading $Z$ boson in simulated events are shown in Fig. 7 for the $2 e 2 \mu$ decay channel. The $4 e, 4 \mu$ decay channels have similar distributions. This plot leads us to focus on the nominal $\mathrm{Z}$ boson mass region.

After applying Cut-5, the invariant mass distributions for all $\mathrm{Z}$ boson candidates are given in Fig. 8. The corresponding numbers of signal events are given in Table 3.

\section{Results}

To obtain $95 \%$ C.L. limits on the couplings, we apply $\chi^{2}$ criterion without and with a systematic error. The $\chi^{2}$ function is defined as follows: (a)

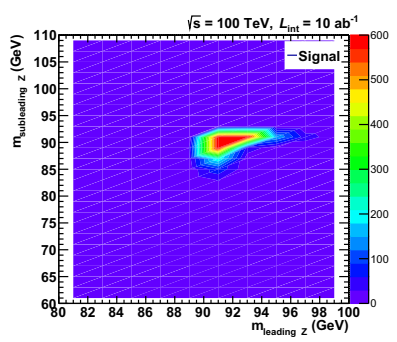

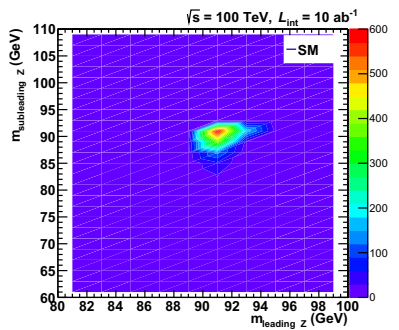

(b)
Fig. 7 Invariant mass distributions of sub-leading $Z$ boson versus leading $Z$ boson for signal (left) and main background (right) for the $2 e 2 \mu$ decay channel. The $\mathrm{z}$-axis corresponds to the number of events where the red color represents the highest number of events

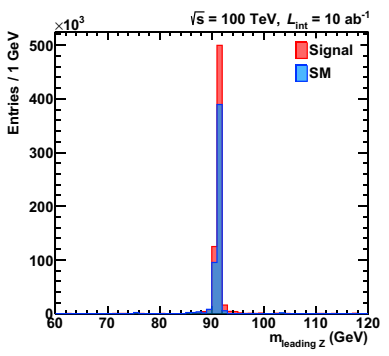

(a)

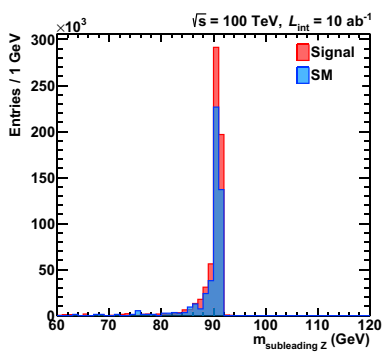

(b)
Fig. 8 Distributions of the reconstructed dilepton candidate mass for four-lepton events selected with both a leading and $\mathbf{b}$ sub-leading onshell $\mathrm{Z}$ bosons

Table 3 The number of events yielded for main background and signal (where all couplings are equal to zero, except $C_{\widetilde{B} W} / \Lambda^{4}=5.0 \mathrm{TeV}^{-4}$ ) of four-lepton events after Cut- 5 shown for each final state and combined at FCC-hh with $\mathcal{L}_{\text {int }}=10 \mathrm{ab}^{-1}$

\begin{tabular}{lllr}
\hline Channel & Signal & Background & \multicolumn{1}{c}{ Total } \\
\hline $4 e$ & 16,308 & 13,991 & 30,299 \\
$4 \mu$ & 32,477 & 26,850 & 59,327 \\
$2 e 2 \mu$ & 76,404 & 71,755 & 148,159 \\
\hline
\end{tabular}

$\chi^{2}=\sum_{i}^{n_{\text {bins }}}\left(\frac{N_{i}^{N P}-N_{i}^{B}}{N_{i}^{B} \Delta_{i}}\right)^{2}$

where $N_{i}^{N P}$ is the total number of events in the existence of effective couplings, $N_{i}^{B}$ is the total number of events of the corresponding SM backgrounds in the $i$ th bin of the invariant mass of the quadruplet-leptons' distribution, and $\Delta_{i}=\sqrt{\delta_{s y s}^{2}+1 / N_{i}^{B}}$ is for the combined systematic $\left(\delta_{\text {sys }}\right)$ and statistical errors in each bin.

The existence of aNTGCs will lead to enhancement of the yield of events at quadruplet-lepton masses. The distributions of the quadruplet-lepton reconstructed mass of events with both leading and sub-leading on-shell $Z$ bosons in the mass range $60-120 \mathrm{GeV}$ for the unified $4 e, 4 \mu$, and $2 e 2 \mu$ channels are depicted in Fig. 9. The limits on probable contributions 


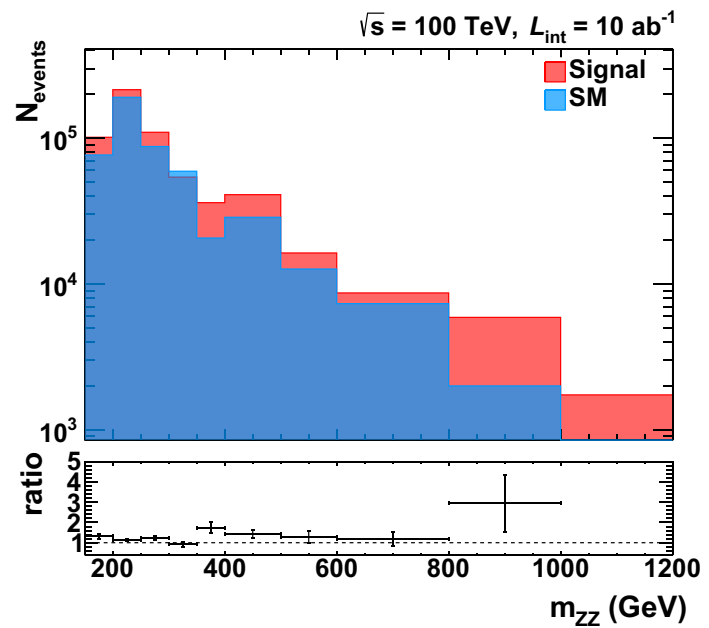

Fig. 9 Distributions of the reconstructed four-lepton invariant mass $m_{Z Z}$. In the $m_{Z Z}$ distribution, the bin contents are normalized to the bin widths. The lower plot shows the ratio of signal and background in the bins. The error bars in the $x$-axis represent the variable bin size in the mass. The error bars in the $y$-axis represents the statistical error in each bin

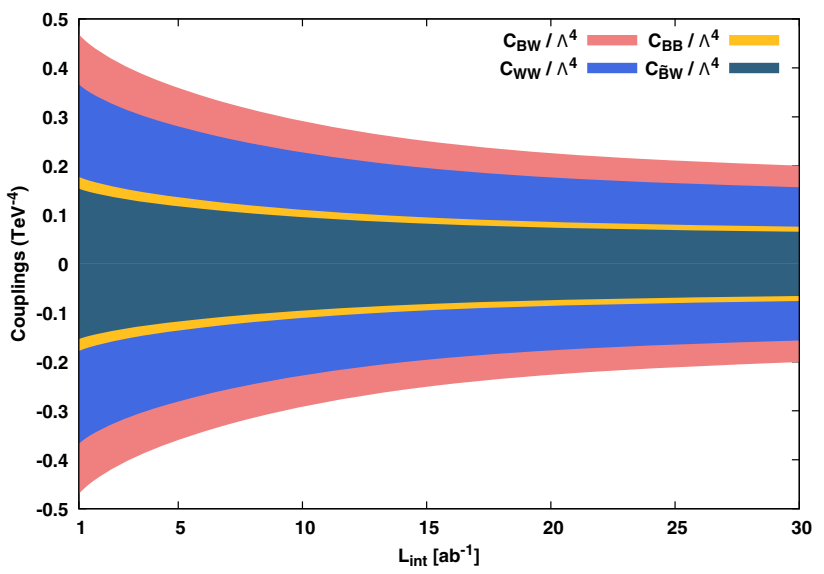

Fig. 10 Estimated sensitivity on aNTG couplings at 95\% C.L. without systematic error as a function of integrated luminosity where there is only one coupling varied at a time from its SM value

from aNTGCs are extracted by using these distributions. In order to check the validity regime of the EFT, we need the minimum coupling value of the coefficients to put the operator scale $\Lambda$ beyond the reach of the kinematical range of the distributions in order not to let the EFT approach break down. The coefficients of the dimension-eight operators could be related to the new physics characteristic scale $\Lambda$ [20]. An upper bound can be put on the new physics scale $\Lambda$ using the fact that the underlying theory is strongly coupled. Assuming couplings $C=O(1)$, we find $\Lambda<\sqrt{4 \pi v \sqrt{s}} \sim 17.5 \mathrm{TeV}$. This upper bound is not violated in this analysis as we have $m_{4 \ell}<1.2 \mathrm{TeV}$ for the kinematic range of invariant mass distributions.

For the analysis of $Z Z$ production with quadruplet-leptons in the final state, we address the number of signal events and one-parameter $\chi^{2}$ results for each coupling varied with integrated luminosity from $1 \mathrm{ab}^{-1}$ to $30 \mathrm{ab}^{-1}$. In the analysis, only one coupling at a time is varied from its $\mathrm{SM}$ value. The results from a $\chi^{2}$ analysis of the couplings describe aTGC interactions of neutral gauge bosons. The coefficients of the operators, denoted by $C_{\tilde{B} W} / \Lambda^{4}, C_{W W} / \Lambda^{4}, C_{B W} / \Lambda^{4}$ and $C_{B B} / \Lambda^{4}$, are given in Fig. 10.

We present the results of one-dimensional 95\% C.L. confidence intervals at $L_{i n t}=10 \mathrm{ab}^{-1}$ under the assumption that any excess in signal over background due exclusively to $C_{\tilde{B} W} / \Lambda^{4}, C_{W W} / \Lambda^{4}, C_{B W} / \Lambda^{4}$ or $C_{B B} / \Lambda^{4}$ are given in Table 4 . We also include the effects of the systematic errors on the limits. The obtained limits without systematic errors are at least one order better than the current limits on these couplings of dim-8 operators converted from the couplings of dim- 6 operators for the process $p p \rightarrow Z Z \rightarrow \ell^{+} \ell^{-} \ell^{\prime+} \ell^{\prime-}$ [14] at the center of mass energy $\sqrt{s}=13 \mathrm{TeV}$ and integrated luminosity $L_{\text {int }}=36.1 \mathrm{fb}^{-1}$ from the LHC.

We find the most sensitive results for CP-conserving operators which contribute to the pair production of neutral electroweak bosons (including $Z Z \gamma$ and $Z Z Z$ vertices). However, the $Z Z \gamma$ and $Z \gamma \gamma$ vertices contribute in the case of the $Z \gamma$ production. Therefore, we expect different sensitivities for the $Z Z$ and $Z \gamma$ productions.

\section{Conclusion and discussion}

In this paper we present a phenomenological cut-based study for probing the limits on the CP-conserving $C_{\tilde{B} W} / \Lambda^{4}$ and CP-violating $C_{W W} / \Lambda^{4}, C_{B W} / \Lambda^{4}$ and $C_{B B} / \Lambda^{4}$ dim-8
Table 4 Estimated one dimensional $95 \%$ C.L. limits on aNTG couplings with and without a systematic error at $L_{\text {int }}=10 \mathrm{ab}^{-1}$

\begin{tabular}{|c|c|c|c|c|}
\hline \multirow{2}{*}{$\begin{array}{l}\text { Couplings } \\
(\mathrm{TeV}-4)\end{array}$} & \multicolumn{4}{|l|}{ Limits at $95 \%$ C.L. } \\
\hline & $\delta_{\text {sys }}=0 \%$ & $\delta_{\text {sys }}=1 \%$ & $\delta_{s y s}=3 \%$ & $\delta_{s y s}=5 \%$ \\
\hline$C_{\tilde{B} W} / \Lambda^{4}$ & {$[-0.09,+0.09]$} & {$[-0.23,+0.23]$} & {$[-0.40,+0.40]$} & {$[-0.51,+0.51]$} \\
\hline$C_{W W} / \Lambda^{4}$ & {$[-0.21,+0.21]$} & {$[-0.60,+0.60]$} & {$[-1.03,+1.03]$} & {$[-1.33,+1.33]$} \\
\hline$C_{B W} / \Lambda^{4}$ & {$[-0.26,+0.26]$} & {$[-0.71,+0.71]$} & {$[-1.23,+1.23]$} & {$[-1.59,+1.59]$} \\
\hline$C_{B B} / \Lambda^{4}$ & {$[-0.10,+0.10]$} & {$[-0.27,+0.26]$} & {$[-0.46,+0.46]$} & {$[-0.59,+0.59]$} \\
\hline
\end{tabular}

For each single anomalous coupling, all parameters other than the one under study are set to zero 
aNTG couplings via $Z Z \rightarrow 4 \ell$ (where $\ell=e$ or $\mu$ ) production at the FCC-hh.

The obtained limits of dim-8 aNTG couplings at $95 \%$ C.L. for $C_{\tilde{B} W} / \Lambda^{4}, C_{W W} / \Lambda^{4}, C_{B W} / \Lambda^{4}$ and $C_{B B} / \Lambda^{4}$ with an $\mathcal{L}_{\text {int }}=10 \mathrm{ab}^{-1}$ are at least one order better than those available prior to this study without systematic error. When we compare these results with the latest search for $v \bar{v} \gamma$ production [22] from the LHC, we have better results on the $C_{\tilde{B} W} / \Lambda^{4}, C_{W W} / \Lambda^{4}$ couplings and improved results on the $C_{B W} / \Lambda^{4}$ and $C_{B B} / \Lambda^{4}$ couplings.

Even with $5 \%$ systematic errors, the obtained bounds for FCC-hh are better than the LHC results on all couplings studied in this paper. The limits of aNTG couplings would benefit from a high luminosity and a high energy when the systematic uncertainties are well reduced below $5 \%$.

Acknowledgements This work was partially supported by the Turkish Atomic Energy Authority (TAEK) under the project Grant no. 2018TAEK(CERN)A5.H6.F2-20.

Data Availability Statement This manuscript has no associated data or the data will not be deposited. [Authors' comment: There is no additional data associated with this paper.]

Open Access This article is licensed under a Creative Commons Attribution 4.0 International License, which permits use, sharing, adaptation, distribution and reproduction in any medium or format, as long as you give appropriate credit to the original author(s) and the source, provide a link to the Creative Commons licence, and indicate if changes were made. The images or other third party material in this article are included in the article's Creative Commons licence, unless indicated otherwise in a credit line to the material. If material is not included in the article's Creative Commons licence and your intended use is not permitted by statutory regulation or exceeds the permitted use, you will need to obtain permission directly from the copyright holder. To view a copy of this licence, visit http://creativecomm ons.org/licenses/by/4.0/.

Funded by SCOAP ${ }^{3}$.

\section{References}

1. M.S. Neubauer, Annu. Rev. Nucl. Part. Sci. 61, 223 (2011). https:// doi.org/10.1146/annurev-nucl-102010-130106

2. D.R. Green, P. Meade, M.-A. Pleier, Rev. Mod. Phys. 89, 035008 (2017). https://doi.org/10.1103/RevModPhys.89.035008. arXiv:1610.07572 [hep-ex]

3. R. Barate et al., (ALEPH), Phys. Lett. B 469, 287 (1999). https:// doi.org/10.1016/S0370-2693(99)01288-5. arXiv:hep-ex/9911003 [hep-ex]

4. M. Acciarri et al., (L3), Phys. Lett. B 465, 363 (1999). arXiv:hep-ex/9909043 [hep-ex]

5. J. Abdallah et al., (DELPHI), Eur. Phys. J. C 30, 447 (2003). https:// doi.org/10.1140/epjc/s2003-01287-0. arXiv:hep-ex/0307050 [hep-ex]

6. G. Abbiendi et al., (OPAL), Eur. Phys. J. C 32,303 (2003). https:// doi.org/10.1140/epjc/s2003-01467-x. arXiv:hep-ex/0310013 [hep-ex]

7. J. Alcaraz et al., (ALEPH, DELPHI, L3, OPAL, LEP Electroweak Working Group) (2006). arXiv:hep-ex/0612034 [hep-ex]
8. S. Schael et al., (ALEPH, DELPHI, L3, OPAL, LEP Electroweak). Phys. Rep. 532, 119 (2013). https://doi.org/10.1016/j.physrep. 2013.07.004. arXiv:1302.3415 [hep-ex]

9. T. Aaltonen et al., (CDF). Phys. Rev. Lett. 108, 101801 (2012). https://doi.org/10.1103/PhysRevLett.108.101801. arXiv: 1112.2978 [hep-ex]

10. T. Aaltonen et al., (CDF Collaboration), Phys. Rev. D 89, 112001 (2014) https://doi.org/10.1103/PhysRevD.89.112001

11. V.M. Abazov et al., (D0). Phys. Rev. Lett. 100, 131801 (2008). $\quad$ https://doi.org/10.1103/PhysRevLett.100.131801. arXiv:0712.0599 [hep-ex]

12. V.M. Abazov et al., (D0). Phys. Rev. D 88, 032008 (2013). https:// doi.org/10.1103/PhysRevD.88.032008. arXiv:1304.5422 [hep-ex]

13. G. Aad et al., (ATLAS). Phys. Rev. Lett. 116, 101801 (2016). https://doi.org/10.1103/PhysRevLett.116.101801. arXiv:1512.05314 [hep-ex]

14. M. Aaboud, G. Aad et al., (ATLAS Collaboration), Phys. Rev. D 97, 032005 (2018). https://doi.org/10.1103/PhysRevD.97.032005

15. A.M. Sirunyan, A. Tumasyan, W. Adam, F. Ambrogi, E. Asilar, T. Bergauer, J. Brandstetter, E. Brondolin, M. Dragicevic et al., Eur. Phys. J. C (2018). https://doi.org/10.1140/epjc/ s10052-018-5567-9

16. A. Senol, Int. J. Mod. Phys. A 29, 1450148 (2014). https://doi.org/ $10.1142 / \mathrm{s} 0217751 \times 14501486$

17. M.L. Mangano, Stand. Theory Part. Phys. (2016). https://doi.org/ 10.1142/9789814733519_0013

18. C. Frye, M. Freytsis, J. Scholtz, M.J. Strassler, J. High Energy Phys. (2016). https://doi.org/10.1007/jhep03(2016)171

19. T. Dorigo, Prog. Part. Nucl. Phys. 100, 211 (2018). https://doi.org/ 10.1016/j.ppnp.2018.01.009

20. A. Senol, H. Denizli, A. Yilmaz, I.T. Cakir, K. Oyulmaz, O. Karadeniz, O. Cakir, Nucl. Phys. B 935, 365 (2018). https://doi.org/10. 1016/j.nuclphysb.2018.08.018

21. C. Degrande, J. High Energy Phys. (2014). https://doi.org/10.1007/ jhep02(2014)101

22. T.A. Collaboration (The ATLAS Collaboration), ATLASCONF 2018, 035 (2018). https://cds.cern.ch/record/2630334/files/ ATLAS-CONF-2018-035.pdf

23. A. Abada, M. Abbrescia, S.S. AbdusSalam, I. Abdyukhanov, J.A. Fernandez, A. Abramov, M. Aburaia, A.O. Acar, P.R. Adzic et al., Eur. Phys. J. C 79 (2019). https://doi.org/10.1140/epjc/ s10052-019-6904-3

24. A. Abada, M. Abbrescia, S.S. AbdusSalam, I. Abdyukhanov, J. Abelleira Fernandez, A. Abramov, M. Aburaia, A.O. Acar, P.R. Adzic et al., Eur. Phys. J. Spec Top 228, 755 (2019). https://doi. org/10.1140/epjst/e2019-900087-0

25. J. Alwall, R. Frederix, S. Frixione, V. Hirschi, F. Maltoni, O. Mattelaer, H.-S. Shao, T. Stelzer, P. Torrielli, M. Zaro, J. High Energy Phys. (2014). https://doi.org/10.1007/jhep07(2014)079

26. T. Sjöstrand, S. Ask, J.R. Christiansen, R. Corke, N. Desai, P. Ilten, S. Mrenna, S. Prestel, C.O. Rasmussen, P.Z. Skands, Comput. Phys. Commun. 191, 159 (2015). https://doi.org/10.1016/j.cpc.2015.01. 024

27. A. Buckley, J. Ferrando, S. Lloyd, K. Nordström, B. Page, M. Rüfenacht, M. Schönherr, G. Watt, Eur. Phys. J. C (2015). https:// doi.org/10.1140/epjc/s10052-015-3318-8

28. R.D. Ball, V. Bertone, S. Carrazza, C.S. Deans, L.D. Debbio, S. Forte, A. Guffanti, N.P. Hartland, J.I. Latorre, J. Rojo, M. Ubiali, Nucl. Phys. B 867, 244 (2013). https://doi.org/10.1016/ j.nuclphysb.2012.10.003

29. J. de Favereau, C. Delaere, P. Demin, A. Giammanco, V. Lemaître, A. Mertens, M. Selvaggi, J. High Energy Phys. (2014). https://doi. org/10.1007/jhep02(2014)057

30. P. Demin. https://cp3.irmp.ucl.ac.be/projects/ExRootAnalysis. Accessed $20 \mathrm{Feb} 2020$ 
31. R. Brun, F. Rademakers, Nuclear instruments and methods in physics research section A: accelerators, spectrometers, detectors and associated equipment. New Comput. Techn. Phys. Res. V 389, 81 (1997). https://doi.org/10.1016/S0168-9002(97)00048-X

32. V. Khachatryan, A. Sirunyan, A. Tumasyan, W. Adam, E. Asilar, T. Bergauer, J. Brandstetter, E. Brondolin, M. Dragicevic, J. Erö et al., J. Instrum. 12, P01020 (2017). https://doi.org/10.1088/1748-0221/ $12 / 01 / \mathrm{p} 01020$
33. M. Tanabashi et al., (Particle Data Group), Phys. Rev. D 98, 030001 (2018). https://doi.org/10.1103/PhysRevD.98.030001

34. U. Baur, D.L. Rainwater, Phys. Rev. D 62, 113011 (2000). https:// doi.org/10.1103/PhysRevD.62.113011. arXiv:hep-ph/0008063 [hep-ph] 